\title{
Management of severe pediatric subglottic stenosis with glottic involvement
}

\author{
Mercy George, MS, Yves Jaquet, MD, Christos Ikonomidis, MD, and Philippe Monnier, MD
}

Objective: We sought to describe our experience in the management of complex glotto-subglottic stenosis in the pediatric age group.

\begin{abstract}
Methods: Between 1978 and 2008, 33 children with glotto-subglottic stenosis underwent partial cricotracheal resection, and they form the focus of this study. They were compared with 67 children with isolated subglottic stenosis (no glottic involvement). The outcomes measured were need for revision open surgical intervention, delayed decannulation (>6 months), and operation-specific and overall decannulation rates. Fisher's exact test was used for comparison of outcomes.
\end{abstract}

Results: Results of preoperative evaluation showed Myer-Cotton grade III or IV stenosis in $32(97 \%)$ patients and grade II stenosis in 1 patient. All patients with glotto-subglottic stenosis were treated with partial cricotracheal resection and simultaneous repair of the glottic pathology. Bilateral fixed vocal cords were seen in $19(58 \%)$ of 33 patients, bilateral restricted abduction was seen in $7(21 \%)$ of 33 patients, and unilateral fixed vocal cord was seen in $7(21 \%)$ of 33 patients. Ten patients underwent single-stage partial cricotracheal resection with excision of interarytenoid scar tissue. The endotracheal tube was kept for a mean period of 7 days as a stent. Twenty-three patients underwent extended partial cricotracheal resection with LT-Mold (Bredam S.A., St. Sulpice, Switzerland) or T-tube stenting. The overall decannulation rate included $26(79 \%)$ patients, and the operation-specific decannulation rate included $20(61 \%)$ patients.

Conclusions: Glotto-subglottic stenosis is a complex laryngeal injury associated with delayed decannulation and decreased overall and operation-specific decannulation rates when compared with those after subglottic stenosis without glottic involvement after partial cricotracheal resection. (J Thorac Cardiovasc Surg 2010;139:411-7)

Stenotic lesions involving the glottis and subglottis can be acquired or congenital in nature. The most common cause of acquired stenosis is postintubation injury. The posterior glottis bears the maximum brunt of the injury because of the posterior displacement of the endotracheal tube by the base of the tongue and the posterior angulation of the trachea. This explains the synchronous involvement of the posterior glottis and of the subglottis in some cases of prolonged intubation. ${ }^{1}$ The majority of the patients with this problem are tracheostomy dependent and have compromised voice production. There is no fixed treatment algorithm possible for the treatment of this challenging problem, and a combination of various surgical techniques becomes necessary as dictated by the extent of the initial injury to achieve decannulation.

There are various surgical techniques that have evolved over the years for the treatment of glotto-subglottic stenosis

\footnotetext{
From the Department of Otolaryngology, Head and Neck Surgery, University Hospital (CHUV), Lausanne, Switzerland.

Disclosures: Philippe Monnier holds financial relationship with the company whose product (LT-Mold) is mentioned in the text.

Received for publication Feb 26, 2009; revisions received April 21, 2009; accepted for publication May 16, 2009; available ahead of print July 2, 2009.

Address for reprints: Mercy George, MS, Department of Otolaryngology, Head and Neck Surgery, Centre Hospitalier Universitaire, Vaudois, Lausanne 1011, Switzerland (E-mail: philippe.monnier@chuv.ch).

$0022-5223 / \$ 36.00$

Copyright (c) 2010 by The American Association for Thoracic Surgery

doi:10.1016/j.jtcvs.2009.05.010
}

(G-SGS). The traditional approach to surgical correction in combined stenosis is usually a laryngotracheal reconstruction (LTR) with anterior and posterior cartilage grafts and stenting through a laryngofissure approach. However, better results are reported in the literature for the treatment of G-SGS by means of cricotracheal resection and synchronous LTR (posterior cricoid split with costal cartilage graft) as a double-stage procedure with long-term stenting in both adults and children., ${ }^{2,3}$ Minimal interarytenoid scarring can be treated as a singlestage procedure with division of the scar tissue and covering of the raw surface with local mucosal flaps or by advancing the posterior membranous trachea with the endotracheal tube kept as a stent for a minimum period of 5 to 7 days. Stenting after reconstruction helps stabilize the posterior cartilage graft and also splints the posterior mucosal flap. We report our experience in the management of severe subglottic stenosis (SGS) with glottic involvement in the pediatric population and review the world literature with regard to this difficult problem. We also describe the operation-specific and overall decannulation rates for severe pediatric SGS with glottic involvement treated at our institution.

\section{MATERIALS AND METHODS}

We obtained approval from the Institutional Review Board at University Hospital, Lausanne, Switzerland, before undertaking this study. Thirty-three $(32 \%)$ of 104 patients extracted from our prospectively collected database between 1978 and 2008 presented with G-SGS. Sixty-seven 


\section{Abbreviations and Acronyms \\ CAA = cricoarytenoid ankylosis \\ G-SGS $=$ glotto-subglottic stenosis \\ LTR = laryngotracheal reconstruction \\ LTS = laryngotracheal stenosis \\ PGS = posterior glottic stenosis \\ SGS $=$ subglottic stenosis}

patients had severe isolated SGS. Four patients who underwent recent operations were excluded from the analysis. All patients underwent diagnostic laryngoscopy and bronchoscopy in the operating room to evaluate the extent of the stenosis with special attention to the cricoarytenoid joint pathology based on protocols reported by us previously in the literature. ${ }^{4}$

Patients with G-SGS were compared with patients with isolated SGS (Table 1). The primary outcome measures were overall and operation-specific decannulation rates. The operation-specific decannulation rate signifies the absence of an additional open procedure after primary surgical intervention to achieve decannulation. We used bivariate analysis with Fisher's exact test for comparing the difference in decannulation rates, delay in decannulation, and revision open surgical intervention between the groups.

\section{RESULTS}

Thirty-three children with combined G-SGS who underwent partial cricotracheal resection (PCTR) with repair of glottic pathology were the focus of this study. Their age ranged from 6 months to 16 years (median, 5 years and 4 months). Stenosis resulted from postintubation injury in 18 $(55 \%)$ patients, external laryngeal trauma in $2(6 \%)$ patients, and idiopathic progressive stenosis in $1(3 \%)$ patient. Mixed subglottic stenosis or acquired on congenital subglottic stenosis was seen in $4(12 \%)$ and $8(24 \%)$ patients, respectively. Thirty-two (97\%) of 33 patients were in MyerCotton grade III $(\mathrm{n}=16)$ or IV $(\mathrm{n}=16)$ stenosis categories. One patient had grade II stenosis with glottic involvement. Most (97\%) of the patients were referred, and 31 (94\%) were tracheotomy dependent. Two (6\%) patients had severe stridor on presentation. Among the 2 patients who presented with stridor, one had stenosis of congenital cause, and the other had acquired postintubation stenosis. Nineteen $(57.5 \%)$ patients had previously failed interventions, which included endoscopic dilatations, laser procedures, LTR, or PCTR. Ten of the 19 patients with prior airway interventions had a combination of the above treatments. The glottic pathology included posterior commissure stenosis with bilateral fixed cords in 19 patients, bilateral restricted abduction of the vocal cords in 7 patients, and unilateral fixed vocal cords in 7 patients (Table 2).

The 67 patients with isolated SGS (no glottic involvement) had the following characteristics. Mean age was 4.3 years (range, 2 months to 14 years). Forty-eight patients had grade III stenosis, 16 had grade IV stenosis, and 3 had grade II stenosis. Eight $(12 \%)$ patients needed revision open surgical intervention, and $6(9 \%)$ patients had delayed decannulation of more than 6 months. Seventy-six percent
(51/67 patients) could be extubated/decannulated within 3 weeks. The overall and operation-specific decannulation rate was $95.5 \%$ and $86.5 \%$, respectively. Based on statistical analysis, children with G-SGS were more likely to have decreased overall and operation-specific decannulation rates when compared with the isolated SGS group $(P=.014$ and .005 , respectively). Also, children with G-SGS showed a tendency toward delayed decannulation that was statistically significant $(P=.002)$. However, significance was not reached for revision open surgical intervention $(P=.541)$ when compared with the isolated SGS group.

\section{Surgical Data and Decannulation Rates in the G-SGS Group}

Ten of 33 patients underwent a single-stage PCTR with interarytenoid scar excision (with free or pedicled mucosal graft), and the endotracheal tube was maintained postoperatively, which acted as a stent. Extended PCTR (PCTR with an additional procedure) was done in 23 patients. The additional procedures included posterior cricoid split with $(\mathrm{n}=18)$ or without $(\mathrm{n}=1)$ a costal cartilage graft and separation of fused vocal cords $(n=4)$. A pedicled flap of the membranous trachea was used to reline the cartilage graft or the denuded cricoid plate. We placed a Montgomery T-tube in 8 patients during the earlier years of our experience, whereas a suprastomal LT-Mold stent (Bredam S.A., St. Sulpice, Switzerland) was preferred in 15 patients during the latter part of our series. ${ }^{5}$ Postoperative endoscopies were performed in all patients, and a majority required several endoscopies (mean, 4.2; range, 1-11), with or without dilatation or granulation tissue removal.

In this series of 33 patients, extubation/decannulation was achieved in 26 patients (overall decannulation rate, 79\%) within a period ranging from 1 week to 3 years (mean, 8 months; median, 3 months). The reason for unsuccessful decannulation was association of comorbidity/syndrome, which included neurologic dysfunction, gastroesophageal reflux disease, and/or extralaryngeal obstruction regardless of a satisfactory postoperative airway $(\mathrm{n}=4)$. One patient with idiopathic stenosis and associated fructosemia had restenosis after an initial satisfactory result. Another child who awaits decannulation had to undergo endoscopic laser epiglottopexy for supraglottic obstruction. A patient with spondyloepiphyseal dysplasia with thoracic cage malformation finally succumbed to the primary pathology before decannulation.

Six $(18 \%)$ patients needed revision open surgical intervention, which included LTR for recurrent posterior glottic stenosis (PGS; $\mathrm{n}=3$ ), revision PCTR for recurrent stenosis $(\mathrm{n}=2)$, and revision anastomosis for partial dehiscence $(n=1)$, before achieving decannulation. The operation-specific decannulation rate therefore was $61 \%$. Three patients required endoscopic laser arytenoidectomy for bilateral residual cricoarytenoid ankylosis (CAA) before successful 
TABLE 1. Comparative profile of patients in the G-SGS and isolated SGS groups

\begin{tabular}{lccc}
\hline $\begin{array}{c}\text { Patient } \\
\text { characteristics }\end{array}$ & $\begin{array}{c}\text { Total } \\
(\mathbf{n = 1 0 0})\end{array}$ & $\begin{array}{c}\text { G-SGS group } \\
(\mathbf{n}=\mathbf{3 3})\end{array}$ & $\begin{array}{c}\text { Isolated SGS } \\
\text { group }(\mathbf{n = 6 7})\end{array}$ \\
\hline Grade II & 4 & 1 & 3 \\
Grade III & 64 & 16 & 48 \\
Grade IV & 32 & 16 & 16 \\
Revision open surgical & 14 & 6 & 8 \\
$\quad$ intervention & & & \\
Decannulation $>6$ mo & 18 & 12 & 6 \\
ND & 10 & 7 & 3 \\
ODR & $90 \%$ & $79 \%$ & $95.5 \%$ \\
OSD & $76 \%$ & $61 \%$ & $86.5 \%$ \\
\hline$G-S G S$, Glotto-subglottic stenosis; $S G S$, subglottic stenosis; $N D$, not decannulated; \\
ODR, overall decannulation rate; $O S D$, operation-specific decannulation rate.
\end{tabular}

decannulation. There were no iatrogenic recurrent laryngeal nerve injuries.

Preoperative evaluation of voice was not possible because the majority of these children were tracheostomized (31/33 patients) or aphonic on presentation. Postoperative voice was mostly dependent on the initial vocal cord pathology with moderate-to-severe dysphonia (voice ranged from rough to weak whispering quality) noticed in the majority of the patients with G-SGS. The evaluation focused on both the period before the operation and the patient's condition at the latest examination. The long-term outcome was based on a questionnaire sent to the patients requesting them to rate the symptoms of dyspnea, dysphonia, and dysphagia. The follow-up period ranged from 6 months to 23 years (mean, 4 years and 3 months). Of the twenty-four patients available for postoperative follow-up examination, $15(62.5 \%)$ had normal breathing, $6(25 \%)$ presented with some exertional dyspnea, and $3(12.5 \%)$ were dyspneic during moderate activity. One patient required a continuous positive airway pressure device during the night. Swallowing was normal in almost all patients, although 2 patients had mild cough during meals. One patient with severe aspiration preoperatively (esophageal atresia) continued to require a nasogastric tube, even after surgical intervention.

\section{Complications}

In our series of 33 pediatric patients with complex G-SGS, there was no intraoperative mortality. Four patients died later of causes unrelated to the operation. Among the 4 deceased patients, 3 could be decannulated after PCTR. The first patient died 19 years after the operation of a drug overdose. The second patient, who had associated congenital malformations and neurologic dysfunction, died at home 6 weeks after the operation of aspiration and severe bronchospasm. The third patient succumbed to congenital cardiac anomalies 3 months after laryngotracheal surgery. The patient with spondyloepiphyseal dysplasia succumbed to the respiratory insufficiency caused by thoracic cage malforma-
TABLE 2. Patient data in the G-SGS group $(n=33)$

\begin{tabular}{lrc}
\hline & No. & $\%$ \\
\hline Cause & & \\
Acquired & 20 & 61 \\
Mixed & 8 & 24 \\
Congenital & 4 & 12 \\
IPSS & 1 & 3 \\
Grade & & \\
II & 1 & 3 \\
III & 16 & 48.5 \\
IV & 16 & 48.5 \\
Vocal cord & & \\
Limited abduction & 8 & 24 \\
Unilateral fixation & 5 & 15 \\
Bilateral fixity & 20 & 61 \\
\hline$G$-SGS, Glotto-subglottic stenosis; IPSS, idiopathic progressive subglottic stenosis.
\end{tabular}

tion and respiratory insufficiency despite maintaining the tracheostomy tube postoperatively.

\section{DISCUSSION}

Cause

Acquired laryngotracheal stenosis (LTS) can result from prolonged endotracheal intubation, external laryngeal injury, and caustic or thermal injuries. However, prolonged endotracheal intubation remains the cause of LTS in more than $90 \%$ of patients. ${ }^{6}$ Walner and colleagues ${ }^{7}$ have reported the incidence of SGS in intubated neonates to be between $0.9 \%$ and $2 \%$. It has also been reported that $1 \%$ to $8 \%$ of neonates who have acquired stenosis after intubation will have a congenitally small cricoid. ${ }^{8}$

The management of early postintubation injury is necessary to prevent the formation of cicatricial stenosis. In our series $18(55 \%)$ of 33 patients had G-SGS caused by postintubation injury, $12(36 \%)$ caused by mixed subglottic stenosis or acquired on congenital subglottic stenosis, and 2 $(6 \%)$ caused by external laryngeal trauma, and $1(3 \%)$ patient had idiopathic progressive stenosis. The extent of stenosis can be worsened in some patients by prior laser treatment, open surgical intervention, or both. In our series of $33 \mathrm{pa}$ tients with G-SGS, $19(58 \%)$ had a history of failed prior endoscopic or open airway intervention. It is also noteworthy that acute external laryngeal injuries, when not meticulously managed, can result in complex LTS. Wound healing caused by fractures, dislocations, mucosal injury, and loss of cartilage support can lead to the development of complete GSGS with a distorted laryngeal framework. This calls for an emergency repair if one wants to avoid the late cicatricial sequelae of this type of injury. The 2 patients in our series with a history of laryngeal trauma came to us after failed attempts at LTR with complete LTS and fusion of the vocal cords.

\section{Surgical Techniques}

G-SGS can be very challenging to treat, and the surgical management of this problem has therefore evolved over the 

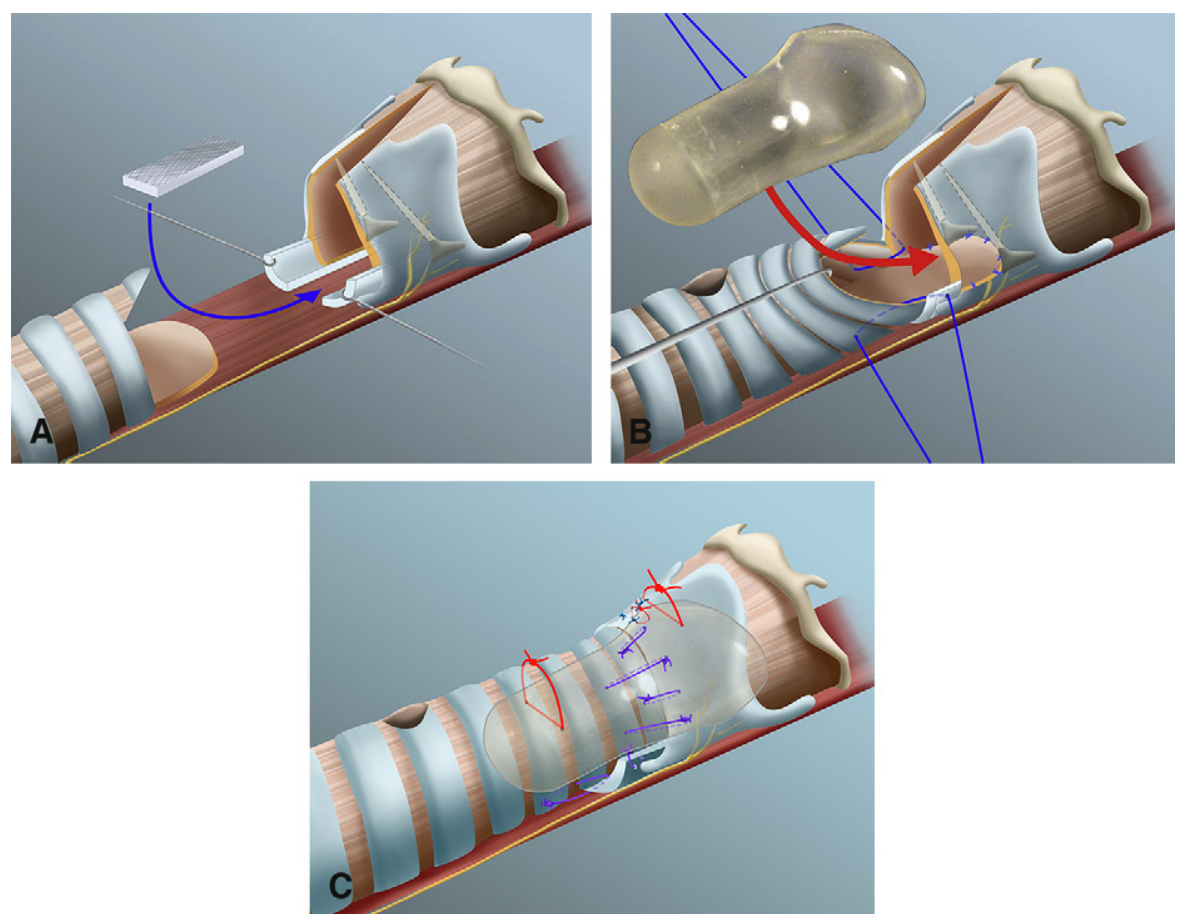

FIGURE 1. Extended partial cricotracheal resection for the cure of combined subglottic and posterior glottic stenosis. A, After conventional partial cricotracheal resection, a posterior cricoid split is performed through a temporary thyrotomy, and a costal cartilage graft is interposed between the 2 parts of the posterior cricoid (arrow). B, The trachea is advanced upward, and its membranous portion is sutured to the mucosa of the posterior commissure of the larynx covering the costal cartilage graft, followed by stenting with an LT-Mold (arrow). C, The completed anastomosis and LT-Mold are fixed with Prolene sutures (Ethicon, Inc, Somerville, NJ).

years. Bogdsarian and Olsen ${ }^{9}$ classified PGS after analyzing their experience with 10 patients. Their classification, based on the severity of scar formation and arytenoid cartilage fixation, is useful for prognostication about and comparison of different treatment results for patients with this difficult problem. Type 1 stenosis is a scar band between the vocal folds that is anterior and is separate from the posterior commissural area. Type II stenoses involve the mucosa, musculature, or both of the commissure. Type III and IV stenoses are defined by unilateral and bilateral cricoarytenoid joint fixation, respectively. Numerous endoscopic and open surgical approaches have been described for the management of G-SGS. Overenthusiastic use of laser treatment, dilatation, and/or stenting to treat this problem can be disastrous, with worsening of the initial condition. According to Simpson and associates, ${ }^{10}$ the indications for use of laser treatment should be limited to a noncircumferential scar or a circular thin web without cartilaginous collapse. However, the laser can be used for arytenoidectomy or posterior cordotomy in case of bilateral vocal cord paralysis. The failure to address cricoarytenoid joint function in complex stenoses results in delayed or unsuccessful decannulation. Combined laryngotracheal injuries and PGS with arytenoid fixation are not suitable for solely endoscopic laser treatment. ${ }^{10,11}$ We used a laser in 6 of our patients as an ancillary treatment for removal of granulations $(n=4)$ and arytenoidectomy for $\mathrm{CAA}(\mathrm{n}=3)$ to improve the interarytenoid distance after the open operation.
When SGS is associated with cicatricial interarytenoid adhesion, PCTR with simple excision of the scar is effective. ${ }^{12}$ When scar tissue involves the posterior commissural area, adequate mucosal cover after the excision of the scar is important to prevent restenosis. ${ }^{13}$ When free movement of the cricoarytenoid joint is not possible, an expansion LTR with cartilage grafting has been recommended in the pediatric population. ${ }^{14,15}$ The disadvantage here is the unsteadiness and distortion of the laryngeal framework caused by the laryngofissure with combined anterior and posterior costal cartilage grafts. Younis and Lazar ${ }^{16}$ reported an overall decannulation rate of $83 \%$ in a group of 46 patients who underwent single-stage LTR with SGS. Only 4 patients in this series had combined PGS and SGS; another 4 patients had bilateral vocal cord paralysis. In their series vocal cord fixation, interarytenoid stenosis, and glottic stenosis were predictive factors affecting failure of single-stage LTR.

Extended PCTR with posterior cricoid split and costal cartilage grafting provides better results than LTR with anterior and posterior cartilage grafts. In extended PCTR the pedicled flap of membranous trachea used to reline the cartilage graft provides a fully mucosalized subglottic airway (Figure 1). The steadiness of the tracheal ring used for the thyrotracheal anastomosis explains the better success rate when compared with LTR. In the Toronto General series of 29 adult patients with G-SGS treated with extended PCTR, 23 (79\%) could be successfully decannulated. ${ }^{17}$ The results of extended 


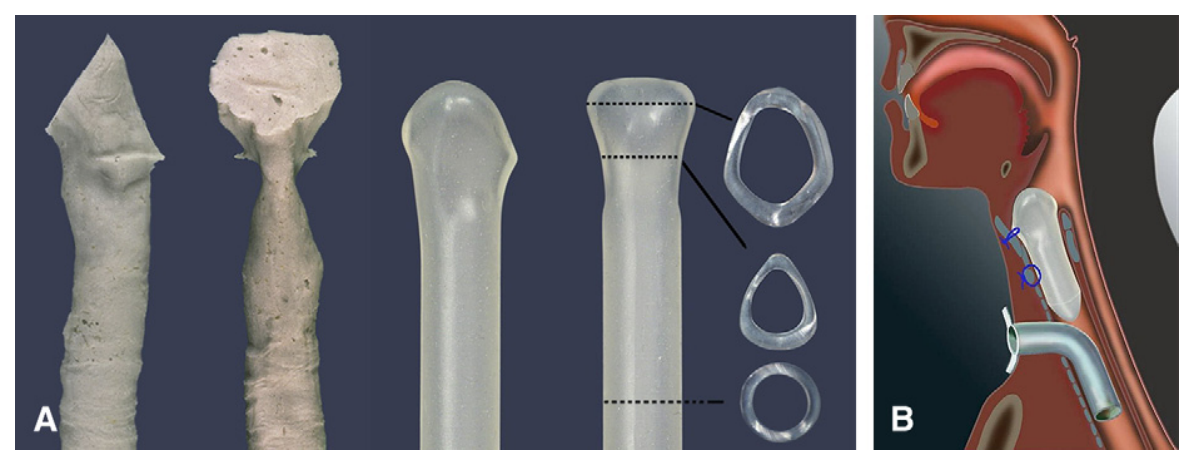

FIGURE 2. A, Design of the LT-Mold, with cross-sections at different levels of the prosthesis shown on the right side. Adult and pediatric molds of cadaver larynges were used to design the LT-Mold. The interarytenoid distance has been increased to that of a fully abducted larynx. B, The LT-Mold is cut at the appropriate length to fit in to the reconstructed suprastomal airway. It is then fixed to the larynx and trachea with 3-0 Prolene sutures.

PCTR done for complex pediatric LTS were found to be less favorable than those of simple PCTR done for isolated SGS in the previous reports published in the literature. The decannulation rate was $98 \%$ and $100 \%$ for grade III to IV isolated SGS in the earlier case series of Lausanne and Cincinnati, respectively. ${ }^{18-20}$ The association of glottic involvement showed a decrease in the overall decannulation rate to $79 \%$ in this group of 33 patients when compared with the isolated stenosis group $(P=.014)$. We also noticed that the time to decannulation in patients with G-SGS was increased (mean, 8 months) when compared with that in patients with isolated SGS. Fifty-one (76\%) of 67 patients with isolated SGS could be extubated within 3 weeks after PCTR.

Inglis and coworkers ${ }^{21}$ have reported their experience with endoscopic posterior cricoid split and rib graft insertion in 10 children with PGS and SGS. They found this approach to be effective in selected cases of PGS with mild-to-moderate SGS. The results of this approach were not satisfactory when performed in patients with grade III to IV SGS. Thome and coworkers ${ }^{22}$ have described the use of a buccal mucosal graft for posterior cricoid splitting for SGS, PGS, or both in 60 patients (45 adults and 15 children) with a $90 \%$ decannulation rate. The graft uptake was $100 \%$, and the subglottic remodeling was also successful with this reconstruction.

Similar to evidence in the literature concerning the postoperative reversal of pseudoparalysis of the vocal cords caused by the CAA, we also noticed an improvement in vocal cord function either partially or completely after surgical intervention. ${ }^{23}$ Ninety-seven percent of the patients in this series had grade III or IV stenoses, with either 1 or 2 ankylosed cricoarytenoid joints in the majority. It is certainly worthwhile to deliberately open the joint during the operation to release adhesions and fibrosis in CAA. Based on our experience, the key to a successful outcome seems to be stabilization of the posterior cartilage graft, lining of the cricoarytenoid joint with the pedicled flap of membranous trachea, and maintenance of the arytenoid cartilage in the abducted position with the use of proper stenting. There are also reports in the literature about the return of vocal cord mobility in patients with CAA by means of lysis of adhesions at the medial aspect of the cricoarytenoid joint and maintenance of the arytenoids stented in abduction. Vocal cord mobility returned in 9 of 10 patients treated in this manner. ${ }^{24,25}$

\section{Stenting}

The use of the silicone LT-Mold significantly reduces the incidence of granulation tissue formation, and it can be left in place for several months in more difficult cases of G-SGS (Figure 2). The only prerequisite is the presence of a tracheostoma. If excessive granulation tissue is encountered on the cartilage graft after removing the LT-Mold, application of mitomycin $\mathrm{C}$ (after removing the granulations) and reinsertion of the LT-Mold for an additional period of 1 or 2 months will be effective for successful decannulation. We used the Montgomery T-tube in 8 patients in the earlier part of the series. Although the T-tube can be effective for stenting a purely tracheal stenosis, it is not ideal for stenting complex G-SGS. ${ }^{5}$ Furthermore, it is not optimal for restoring a sharp anterior commissure of the larynx in cases of synechiae of the vocal cords, and it can induce erosions and granulation tissue on the ventricular bands and on the epiglottis.

Stern and associates ${ }^{26}$ have reported that stenting with a T-tube is relatively safe in children, but the problem of aspiration was seen in $11.5 \%$ and granulation tissue was seen in $16 \%$ of the 26 patients published in this series. Hoasjoe and colleagues, ${ }^{13}$ in their series of 10 patients, have described the use of an autologous graft (full-thickness skin graft or perichondrocutaneous graft) in the interarytenoid area after laryngofissure with a Montgomery stent for the management of isolated PGS. However, 1 patient had to undergo reopening of the laryngofissure for removal of the stent because it was fractured.

\section{Postoperative Course and Outcome}

The postoperative course and decannulation time were influenced by many factors, which included the complexity of the stenosis, vocal cord mobility, and associated comorbidities. The mean number of postoperative endoscopies 

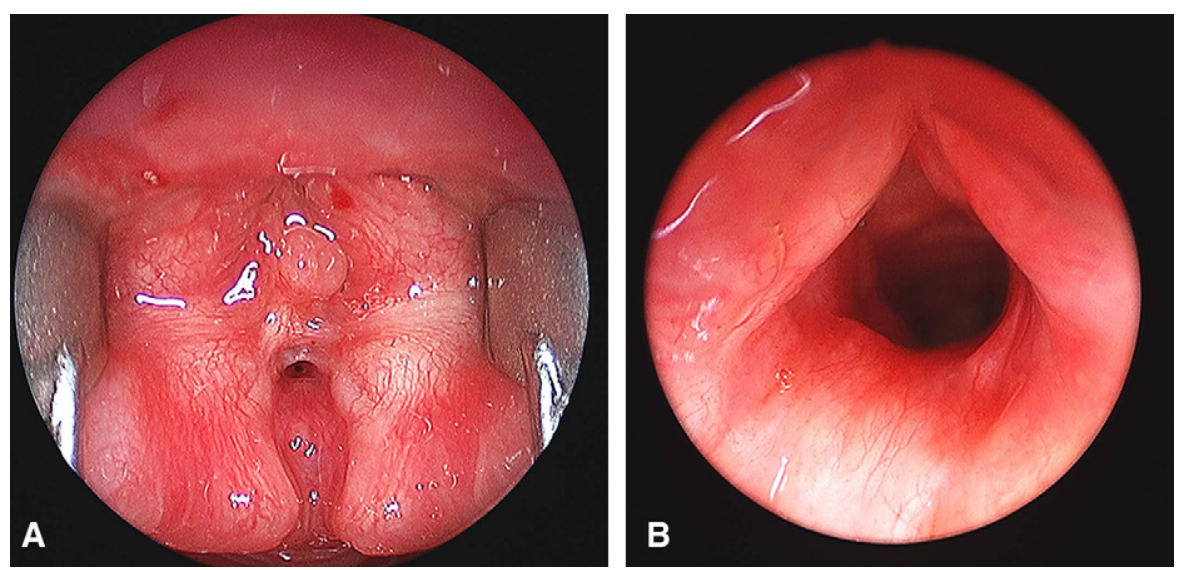

FIGURE 3. A, Preoperative endoscopic picture of a 1-year-old boy who presented with mixed stenosis (acquired on congenital) glotto-subglottic stenosis (vocal cord fusion with posterior pinhole opening and grade III subglottic stenosis). B, Postoperative endoscopic view after 6 months of synchronous partial cricotracheal resection and laryngotracheal reconstruction showing patent airway with restoration of a satisfactory triangular glottis.

was increased to 4.2 (range, 1-11) in this series of patients with G-SGS when compared with a mean of 2.5 (range, $1-7)$ endoscopies in the isolated SGS group. One patient in our series with a history of external trauma to the larynx and prior airway interventions sustained an erosion of the lower part of the thyroid cartilage caused by high tracheostomy. He underwent PCTR extending almost up to the level of the vocal cords with removal of scar tissue at the glottic level and reconstruction of the glottis with the false vocal cords. No stenting was done initially, but 2 weeks postoperatively, a silicone tube was inserted endoscopically with the Lichtenberger needle carrier. The stent was removed 1 month later with a good outcome. The patient is 22 years old now, and the postoperative follow-up period is 9 years. His airway is stable with no aspiration, but he has a breathy voice.

Decannulation fails in some patients because of causes unrelated to the operation. The association of syndrome or comorbidity can adversely affect the final outcome of decannulation, even after a successful airway reconstruction, which occurred in 4 patients in this series. Revision open surgical intervention was necessary in $18 \%(6 / 33)$ of our patients with G-SGS when compared with $12 \%$ with isolated SGS, although statistical significance was not reached $(P=$ $.541)$. Increased risk of a second open airway procedure associated with vocal cord dysfunction has been noted by White and coworkers. ${ }^{27}$ Thirty-six percent (12/33) of children with G-SGS had delayed decannulation of more than 6 months $(P=.002)$ when compared with $6(9 \%)$ of 67 children in the isolated SGS group. Therefore appropriate counseling of the parents regarding the possibility of a prolonged postoperative course is important when treating a child with G-SGS.

\section{Voice Outcome}

Preoperative evaluation of voice was not possible because the majority of these children were tracheostomized (31/33 patients) or aphonic on presentation. Most of these children referred to us from other countries had severe grade III or IV stenosis $(97 \%)$ with extensive fibrosis and scarring of the vocal cords (Figure 3, A). Some of them $(57.5 \%)$ had a completely distorted larynx with loss of laryngeal framework caused by prior laser treatment or open interventions. We noticed that the postoperative voice quality was directly proportional to the initial extent of the glottic involvement. Long-term voice outcome after PCTR based on the initial pathology is the subject of a prospective study we are carrying out now in patients with LTS. The wide interarytenoid space created by the posterior costal cartilage graft in extended PCTR, a prerequisite for safe decannulation, can adversely affect the voice outcome by preventing complete closure of the vocal cords during phonation (Figure $3, B$ ). ${ }^{20}$

\section{CONCLUSION}

G-SGS is a complex laryngeal injury that necessitates multiple airway procedures depending on the exact nature of the cicatricial lesion and its effect on vocal cord mobility. Therefore it is imperative that the otolaryngologist dealing with this situation should also be well trained in endoscopy and laser treatment in addition to open surgical intervention (PCTR and LTR). G-SGS can also be associated with delayed decannulation and decreased overall and operationspecific decannulation rates when compared with results in a group of patients with isolated SGS. However, by implementing a combination of endoscopic and reconstructive procedures, most patients with complex G-SGS can be decannulated eventually with fewer operations than in the past.

\section{References}

1. Langman AW, Leo KC, Dedo H. The endoscopic Teflon keel for posterior and total glottic stenosis. Laryngoscope. 1989;99:571-7.

2. Maddaus MA, Toth JLR, Gullane PJ, Pearson FC. Subglottic tracheal resection and synchronous laryngeal reconstruction. J Thorac Cardiovasc Surg. 1992; 104:1443-50.

3. Monnier P, Lang F, Savary M. Cricotracheal resection for severe pediatric subglottic stenosis. Int J Pediatr Otorhinolaryngol. 1999;49:283-6. 
4. Monnier P, Lang F, Savary M. Cricotracheal resection for adult and pediatric subglottic stenoses: similarities and differences. Op Tech Otolaryngol Head Neck Surg. 1999;10:311-5.

5. Monnier P. Airway stenting with the LT-mold: Experience in 30 pediatric cases. Int J Pediatr Otorhinolaryngol. 2007;71:1351-9.

6. Cotton RT. Prevention and management of laryngeal stenosis in infants and children. J Pediatr Surg. 1985;20:845-51.

7. Walner DL, Loewen MS, Kimura RE. Neonatal subglottic stenosis-incidence and trends. Laryngoscope. 2001;111:48-51.

8. Mostafa SM. Variation in subglottic size in children. Proc R Soc Med. 1976;69: 793-5.

9. Bogdsarian RS, Olsen NR. Posterior glottic laryngeal stenosis. Otolaryngol Head Neck Surg. 1980;88:765-72.

10. Simpson G, Strong M, Healy G. Predictive factors of success or failure in the endoscopic management of laryngeal and tracheal stenosis by radial incision and dilatation. Ann Otol Laryngol. 1982;91:384-9.

11. Monnier P, George M, Monod ML, Lang F. The role of the CO2 laser in the management of laryngotracheal stenosis: a survey of 100 cases. Eur Arch Otorhinolaryngol. 2005;262:602-8.

12. Mau T, Pletcher SD, Cavangh PW. Minicricothyrotomy approach with fibreoptic guidance for management of posterior glottic stenosis. Laryngoscope. 2007;117: 1488-90.

13. Hoasjoe DK, Franklin SW, Aarstad RF, Day TA, Stucker FJ. Posterior glottic stenosis mechanism and surgical management. Laryngoscope. 1997;107:675-9.

14. Zalzal GH. Posterior glottic fixation in children. Ann Otol Rhinol Laryngol. 1993; 102:680-6.

15. Irwing RM, Bailey CM, Evans JNG. Posterior glottic laryngeal stenosis in children. Int J Pediatr Otorhinolaryngol. 1993;28:11-23.
16. Younis RT, Lazar RH. Laryngotracheal reconstruction with out stenting. Otolaryngol Head Neck Surg. 1997;116:358-62.

17. Pathak I, Gullane P. Management of the posterior commissure and thyrotracheal anastomosis. Op Tech Otolaryngol Head Neck Surg. 1999;10:299-302.

18. Monnier P, Lang F, Savary M. Partial cricotracheal resection for pediatric subglottic stenosis: a single institution's experience in 60 cases. Eur Arch Otolaryngol. 2003;260:295-7.

19. Rutter MJ, Hartley BE, Cotton RT. Cricotracheal resection in children. Arch Otolaryngol Head Neck Surg. 2001;127:289-92.

20. Jaquet Y, Lang F, Pilloud R. Partial cricotracheal resection for pediatric subglottic stenosis: longterm outcome in 57 patients. J Thorac Cardiovasc Surg. 2005;130: 726-32.

21. Inglis AF Jr, Perkins JA, Manning SC, Mouzakes J. Endoscopic posterior cricoid split and rib grafting in 10 children. Laryngoscope. 2003;113:2004-9.

22. Thome R, Thome DC, Behlau M. The use of buccal mucosa graft at posterio cricoid splitting for subglottic stenosis repair. Laryngoscope. 2001;111:2191-4.

23. Rovo L, Venczel k, Torkos A, Majoros V. Endoscopic arytenoid lateropexy for isolated posterior glottic stenosis. Laryngoscope. 2008;118:1550-5.

24. Schaefer SD, Close LG, Brown OE. Mobilization of the fixated arytenoid in the stenotic posterior laryngeal commissure. Laryngoscope. 1986;96:656-9.

25. Goodwin WJ Jr, Isaacson G, Kirchner JC, Sasaki CT. Vocal cord mobilisation by posterior laryngoplasty. Laryngoscope. 1988;98:846-8.

26. Stern Y, Willging JP, Cotton RT. Use of Montgomery T-tube in laryngotracheal reconstruction in children: is it safe? Ann Otol Rhinol Laryngol. 1998 107:1006-9.

27. White DR, Cotton RT, Bean JA, Rutter MJ. Pediatric cricotracheal resection, surgical outcomes and risk factor analysis. Arch Otolaryngol Head Neck Surg. 2005;131:896-9. 